\title{
A frequency estimation algorithm based on carrier detection scheme and MUSIC algorithm for DS/BPSK signals
}

\author{
Biwen Wang ${ }^{1}$, Peng Liu ${ }^{2}$, Jiyan Huang ${ }^{1,3}$, Bowu $^{3}$, and Guocai $\mathrm{Mu}^{3}$ \\ ${ }^{1}$ University of Electronic Science and Technology of china (UESTC), Chengdu, China \\ ${ }^{2}$ Space Star Technology Co., Ltd. and State Key Laboratory of Space-Groun Integrated \\ Information Technology, BeiJin, China \\ ${ }^{3}$ Institute of Electronic and Information Engineer In Dongguan Uestc, Dongguan, China \\ 1'shinhwachengzi@126.com, 1huangjiyan@uestc.edu.cn, ${ }^{21}$ 1p1381074@163.com
}

Keywords: direct sequence spread spectrum signal; frequency estimation; carrier detection; MUSIC;

\begin{abstract}
This paper presents a frequency estimator based on carrier detection scheme and MUSIC algorithm which can be used for a BPSK direct sequences spread spectrum signals systems. Under non-cooperative circumstance, the nonlinear carrier detection method is used to detect the presence of DS signal, and it created detectable components of the multiplies of the carrier frequency .Combined with MUSIC algorithm, the carrier frequency can be accurate estimated without the prior knowledge of received signals. The theory of proposed algorithm is introduced in this paper and simulation results have shown that the algorithm has good estimation performance even when signals are buried in strong background noise.
\end{abstract}

\section{Introduction}

Direct sequence spread spectrum communication has useful properties of broad bandwidth, low power spectrum density, low probability of interception and detection. Therefore, it is widely used in mobile communication, modern military communication, and C3I systems. Since the power spectrum of DS signals distributes in a broad frequency band, it is difficult to detect the signals. The ordinary detection methods, like energy detection method [1], can 't meet the practical requirements for the detection of these signals. Cyclic spectrum method can accurately estimate the carrier frequency, but it is require large amount of computation[2]. To meet the needs of frequency estimation precision and low computational amount, combined with characteristics of nonlinear carrier detection and MUSIC algorithm, an algorithm is proposed. The nonlinear carrier detection method [3]produce carrier harmonics $2 f_{0}$ for DS/BPSK signals, and MUSIC algorithm[4] based on the orthogonality of the signal subspace and noise subspace to estimate harmonics frequency. This paper introduced the theory of proposed algorithm and simulation results have shown that it has good estimation performance even in low SNR background.

\section{Theory}

I.Carrier detection scheme

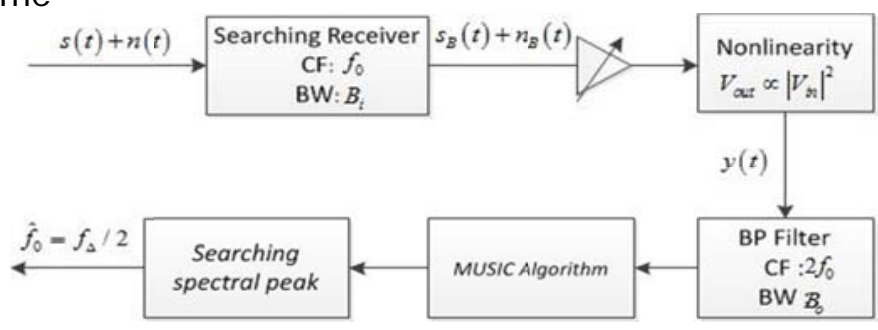

Figure 1 Nonlinear carrier detection system

The nonlinear carrier detection system used in this algorithm is presented in Fig 1.

The DS signal, together with Gaussian white noise, is passed through a searching receiver The signal and noise at the output of the bandpass filter are denoted by $s_{B}(t)$ and $n_{B i}(t)$, respectively. 
After nonlinear transform, the resulting combination of signal and noise is passed through a bandpass filter. The output is denoted by $y(t)$.

The general DS/BPSK signal of interest at the input is[5]

$$
s(t)=\sqrt{2 P_{s}} d(t) c(t) \cos \left(2 \pi f_{0} t+\varphi\right)
$$

In which $f_{0}$ is the estimated carrier frequency, $P_{s}$ is the signal power of DS signal and $\varphi_{0}$ is a random phase variable uniformly distributed on $[-\pi, \pi] . d(t)$ and $c(t)$ are the baseband message signal and PN signal.

Because the center frequency of first searching receiver, we can obtain $s_{B}(t) \approx s(t)$ and $n_{B}(t)$ is narrowband Gaussian white noise, $n_{B}(t): N\left(0, \sigma_{n}^{2}\right)$, the noise power is $B_{i} N_{0}$.After the nonlinear transform, the signal and noise is

$$
y(t)=\left[s(t)+n_{B}(t)\right]^{2}=s^{2}(t)+2 s(t) n_{B}(t)+n_{B}^{2}(t)
$$

Under the condition of low SNR, the cross-component $s_{B}(t)$ and $n_{B}(t)$ can be neglected. The sine wave component is

$$
\begin{aligned}
s^{2}(t) & =2 P_{s} d^{2}(t) c^{2}(t) \cos ^{2}\left(2 \pi f_{0} t+\varphi\right)=2 P_{s} \cos ^{2}\left(2 \pi f_{0} t+\varphi\right) \\
& =P_{s} \cos \left(4 \pi f_{0} t+2 \varphi\right)+P_{s}
\end{aligned}
$$

Hence,

$$
y(t)=P_{s} \cos \left(4 \pi f_{0} t+2 \varphi\right)+P_{s}+n_{B}^{2}(t)=P_{s} \cos \left(4 \pi f_{0} t+2 \varphi\right)+P_{s}+z(t)
$$

Let $n_{B}^{2}(t)$ is $z(t)$.Referring to [6], the noise $z(t)$ has a central chi-square distribution with mean $\sigma_{n}^{2}$ and variance $2 \sigma_{n}^{4}$. Omitting the zero frequency component of $y(t)$, we can obtain

$$
y(t)=P_{s} \cos \left(4 \pi f_{0} t+2 \varphi\right)+z(t)
$$

\section{II.MUSIC algorithm}

In the form of vector to express signal, it is composed of $M$ sampling values

$$
\begin{aligned}
y(n) & =[y(n) \mathrm{L} y(n+M-1)]^{T}=A x(n)+z(n) \\
& =\left[\begin{array}{cccc}
e^{-j \omega_{1}} & 1 & \mathrm{~L} & 1 \\
\mathrm{M} & e^{-j \omega_{2}} & \mathrm{~L} & e^{-j \omega_{K}} \\
e^{-j(M-1) \omega_{1}} & e^{-j(M-1) \omega_{2}} & \mathrm{~L} & e^{-j(M-1) \omega_{K}}
\end{array}\right]\left[\begin{array}{c}
\alpha_{1} e^{j\left(\omega_{1} n+\varphi_{1}\right)} \\
\alpha_{2} e^{j\left(\omega_{2} n+\varphi_{2}\right)} \\
\mathrm{M} \\
\alpha_{K} e^{j\left(\omega_{K} n+\varphi_{K}\right)}
\end{array}\right]+\left[\begin{array}{c}
z(n) \\
z(n+1) \\
\mathrm{M} \\
z(n+2)
\end{array}\right]
\end{aligned}
$$

where the $A x(n)$ and $z(n)=v^{2}(n)$ are double carrier frequency component and noise component. $v(n)$ denoted the additive Gaussian white noise.

Using the fact $\left.E\left\{v^{2}(n) v(n-m)\right\}\right]=E\left\{v(n) v^{2}(n-m)\right\}=0$

So the autocorrelation function of $z(n)$ is

$$
\begin{aligned}
r_{z}(m) & =E\{z(n) z(n-m)\}]=E\left\{v^{2}(n) v^{2}(n-m)\right\} \\
& =E\left\{v^{2}(n)\right\} E\left\{v^{2}(n-m)\right\}+2 E\{v(n) v(n-m)\} E\{v(n) v(n-m)\} \\
& =r_{v}^{2}(0)+2 r_{v}^{2}(m)= \begin{cases}3 \sigma_{n}^{4} & m=0 \\
\sigma_{n}^{4} & m \neq 0\end{cases}
\end{aligned}
$$

Hence the autocorrelation matrix of $z(n)$ is

$$
\left.R_{z}=E\left\{z(n) z^{H}(n)\right\}\right]=E\left\{v^{2}(n)\left[v^{2}(n)\right]^{H}\right\}=\left[\begin{array}{cccc}
3 \sigma_{n}^{4} & \sigma_{n}^{4} & \mathrm{~L} & \sigma_{n}^{4} \\
\sigma_{n}^{4} & 3 \sigma_{n}^{4} & \mathrm{~L} & \sigma_{n}^{4} \\
\mathrm{M} & & \mathrm{O} & \mathrm{M} \\
\sigma_{n}^{4} & \sigma_{n}^{4} & \mathrm{~K} & 3 \sigma_{n}^{4}
\end{array}\right]
$$

Consider the autocorrelation matrix of $y(n)$ is 


$$
\begin{aligned}
& \left.R_{y}=E\left\{y(n) y^{H}(n)\right\}\right]=E\left\{[A x(n)+z(n)][A x(n)+z(n)]^{H}\right\} \\
& =A P A^{H}+R_{z}=A P A^{H}+C+3 \sigma_{n}^{2} I \\
& \text { Where } \quad C=\left[\begin{array}{cccc}
0 & \sigma_{n}^{4} & \mathrm{~L} & \sigma_{n}^{4} \\
\sigma_{n}^{4} & 0 & \mathrm{~L} & \sigma_{n}^{4} \\
\mathrm{M} & & \mathrm{O} & \mathrm{M} \\
\sigma_{n}^{4} & \sigma_{n}^{4} & \mathrm{~K} & 0
\end{array}\right]
\end{aligned}
$$

Characteristic decomposition of $R_{y}$ is

$$
R_{y}=U \Sigma U^{H}=\left[U_{s}, U_{n}\right]\left[\begin{array}{cc}
\Sigma^{\prime} & 0 \\
0 & 3 \sigma_{n}^{4} I_{M-K}
\end{array}\right]\left[\begin{array}{l}
U_{s}^{H} \\
U_{n}^{H}
\end{array}\right]
$$

Where $\Sigma^{\prime}$ contains $\mathrm{K}$ large eigenvalues, $U_{s}$ is the signal subspace, $U_{n}$ is the noise subspace.

Owing to the signal is passed through bandpass filter, the frequency range is its bandwidth. So there is no need to search spectral peak in the full range frequency domain. Also we can choose appropriate frequency resolution to improve estimate performance. The frequency vector is

$$
\alpha(\omega)=\left[\begin{array}{llll}
1 & e^{-j \omega} & \mathrm{L} & e^{-j(M-1) \omega}
\end{array}\right]^{T} \quad \omega \in 2 \pi *\left[2 f-B_{o} / 2,2 f+B_{o} / 2\right]
$$

In which, $f$ is the centre frequency of bandpass filter.

Pseudospectra of MUSIC algorithm can be constructed as

$$
P(\omega)=\frac{1}{\alpha^{H}(\omega) U_{n} U_{n}^{H} \alpha(\omega)}
$$

Then searching the Pseudospectra peak, find out the maximum of $P(\omega)$, the peak position is $f_{\Delta}$. So we can obtain the estimation frequency $\hat{f}_{0}=f_{\Delta} / 2$.

\section{Simulation}

Several experiments were made to test the performance of estimator proposed above. The power spectral density of nonlinear carrier detection scheme when the SNR equaling, $-16 d B$ was shown in Fig3 .The line spectrum at the harmonics of carrier frequency was clear, so we can get the correct evaluated results. With the drops of SNR, signal was buried in strong background noise and the definition line spectrum was decreased. As shown in Fig 4 when SNR equals $-22 d B$.

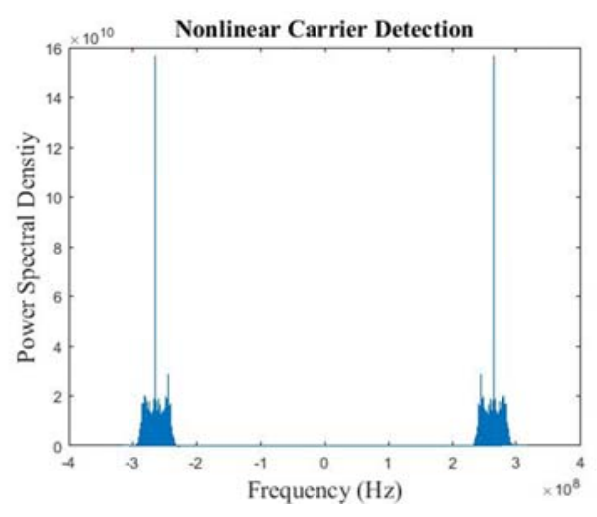

Figure 2 Power spectral density at $\mathrm{SNR}=-16 \mathrm{~dB}$

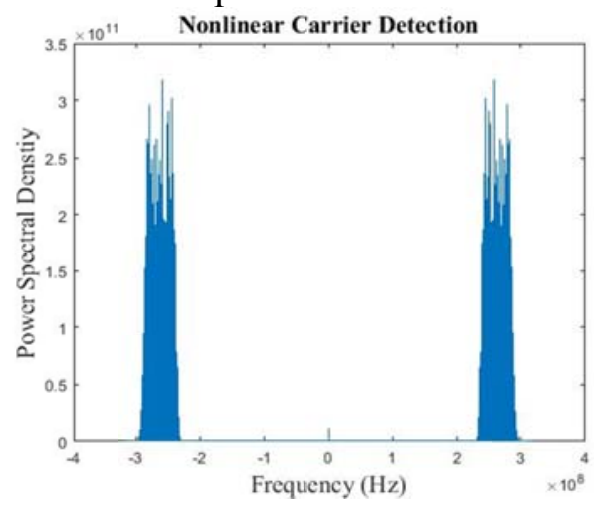

Figure 3 Power spectral density at $\mathrm{SNR}=-22 \mathrm{~dB}$

Then we use MUSIC algorithm to estimate harmonics frequency. Fig 5 shows the estimation errors of FFT and MUSIC under different SNR environments. The MUSIC algorithm have good performance. 


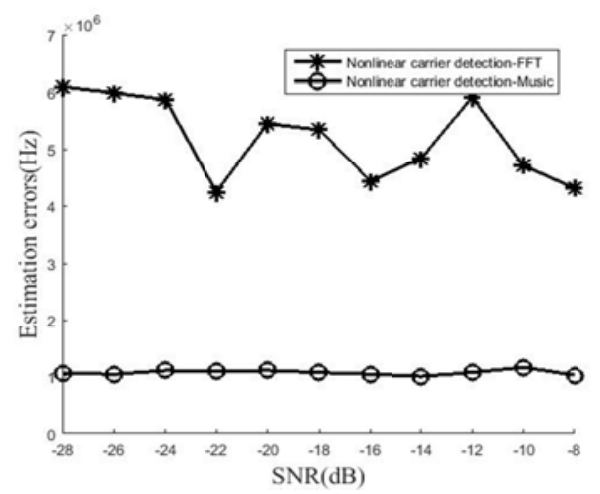

Figure 4 Estimation errors of FFT and MUSIC

From the above figures, we know that with the drop of SNR , the performance of carrier detector deteriorated. But after MUSIC, the carrier frequency can be easily extracted by searching the spectral peaks. The accurate estimation is get then.

\section{Conclusion}

Making use of MUSIC orthogonality of the signal subspace and noise subspace, we can detect the sine wave with double carrier frequency from the output of nonlinear transform of DSSS signal carrier detector. Because the frequency search domain is defined, we can choose appropriate frequency resolution to obtain better detection performance. The algorithm has good estimation performance and low computational complexity even when signals are buried in strong background noise.

\section{Acknowledgements}

This work was supported by the Open Research Fund of State Key Laboratory of Space-Ground Integrated Information Technology under grant No.2014_CXJJ-DH_09,Guangdong Provincial Natural Science Foundation of china (2015A030313821), and Dongguan city social science and technology development program (2013108101002).

\section{Reference}

[1] H.Urkowitz, "Energy Detection of Unknown Deterministic Signal", Proc. JEFF,Vol.55, pp. 523-531.1967.

[2] W. A. Gardner The spectral correlation theory of cyclostationary time-series, IEEE Signal Processing, 1986

[3] D. A. Hill and J. B. Bodie, "Experimental carrier detection of BPSK and QPSK direct sequence spread spectrum signals," in Conf. Rec.MILCOM'95, vol. 1, Nov. 1995, pp. 362-367.

[4] Stoica P, Nehorai A. MUSIC ,maximum likelihood, and Cramer-Rao bound[M]. IEEE Trans.on ASSP , $1989,37(5): 720 \sim 741$.

[5]Theory of Spread-Spectrum Communications-A Tutorial IEEE TRANSACTIONS ON COMMUNICATIONS, VONLCO.O . M -30, 5, MAY 1982

[6]Proakis J G. Digital Communications, 3rd Edition[J]. MA : McGraw-Hill, 2001. 